\title{
Second Harmonic Imaging Microscopy
}

Leslie M. Loew, ${ }^{*}$ Andrew C. Millard, ${ }^{*}$ Paul J. Campagnola, ${ }^{*}$ William A. Mohler, ${ }^{*}$ and Aaron Lewis ${ }^{\ddagger}$

* Center for Biomedical Imaging Technology, University of Connecticut Health Center, Farmington, CT 06030-1507 USA

Division of Applied Physics, Hebrew University of Jerusalem, Jerusalem 91904, Israel

Second Harmonic Generation (SHG) has been developed in our laboratories as a high- resolution non-linear optical imaging microscopy ("SHIM") for cellular membranes and intact tissues. SHG is a non-linear process that produces a frequency doubling of the intense laser field impinging on a material with a high second order susceptibility. It shares many of the advantageous features for microscopy of another more established non-linear optical technique: two-photon excited fluorescence (TPEF). Both are capable of optical sectioning to produce 3D images of thick specimens and both result in less photodamage to living tissue than confocal microscopy. SHG is complementary to TPEF in that it uses a different contrast mechanism and is most easily detected in the transmitted light optical path. It also does not arise via photon emission from molecular excited states, as do both 1- and 2-photon excited fluorescence. SHG of intrinsic highly ordered biological structures such as collagen has been known for some time but only recently has the full potential of high resolution 3D SHIM been demonstrated on live cells and tissues. For example, Figure 1 shows SHIM from microtubules in a living organism, $C$. elegans. The images were obtained from a transgenic nematode that expresses a $\beta$-tubulin-green fluorescent protein fusion and Figure 1 also shows the TPEF image from this molecule for comparison. In addition, we have developed molecular probes based on the styryl class of fluorescent chromophores that show large resonance-enhanced SHG signals when bound to membranes. The large charge redistributions between the ground and excited states in these dyes are the basis of the resonanceenhancement mechanism that enables SHIM of cell membranes. Figure 2 displays SHIM and TPEF micrographs of a neuronal cell stained with a styryl dye that has an appended chiral sugar moiety; we find that the covalent linkage of a chiral group to the chromophores can enhance the SHG signal by as much as a factor of 2 . The styryl dyes have been developed as electrochromic indicators of membrane potential and have seen extensive use for spatiotemporal mapping of electrical activity by fluorescence microscope imaging. Interestingly, the SHG signals from these dyes show even greater sensitivity to potential than the fluorescence signal. Thus, SHIM promises to be a potent new microscope imaging modality for the examination of structure and function in living cells.

\section{Recent Reviews}

Loew LM, Campagnola P, Lewis A, Wuskell JP. 2002. Confocal and nonlinear optical imaging of potentiometric dyes. Methods Cell Biol 70:429-52.

Millard AC, Campagnola PJ, Mohler W, Lewis A, Loew LM. 2003. Second harmonic imaging microscopy. In: Marriott G, Parker I, editors. Methods in Enzymology. San Diego: Academic Press. p 47-69. 

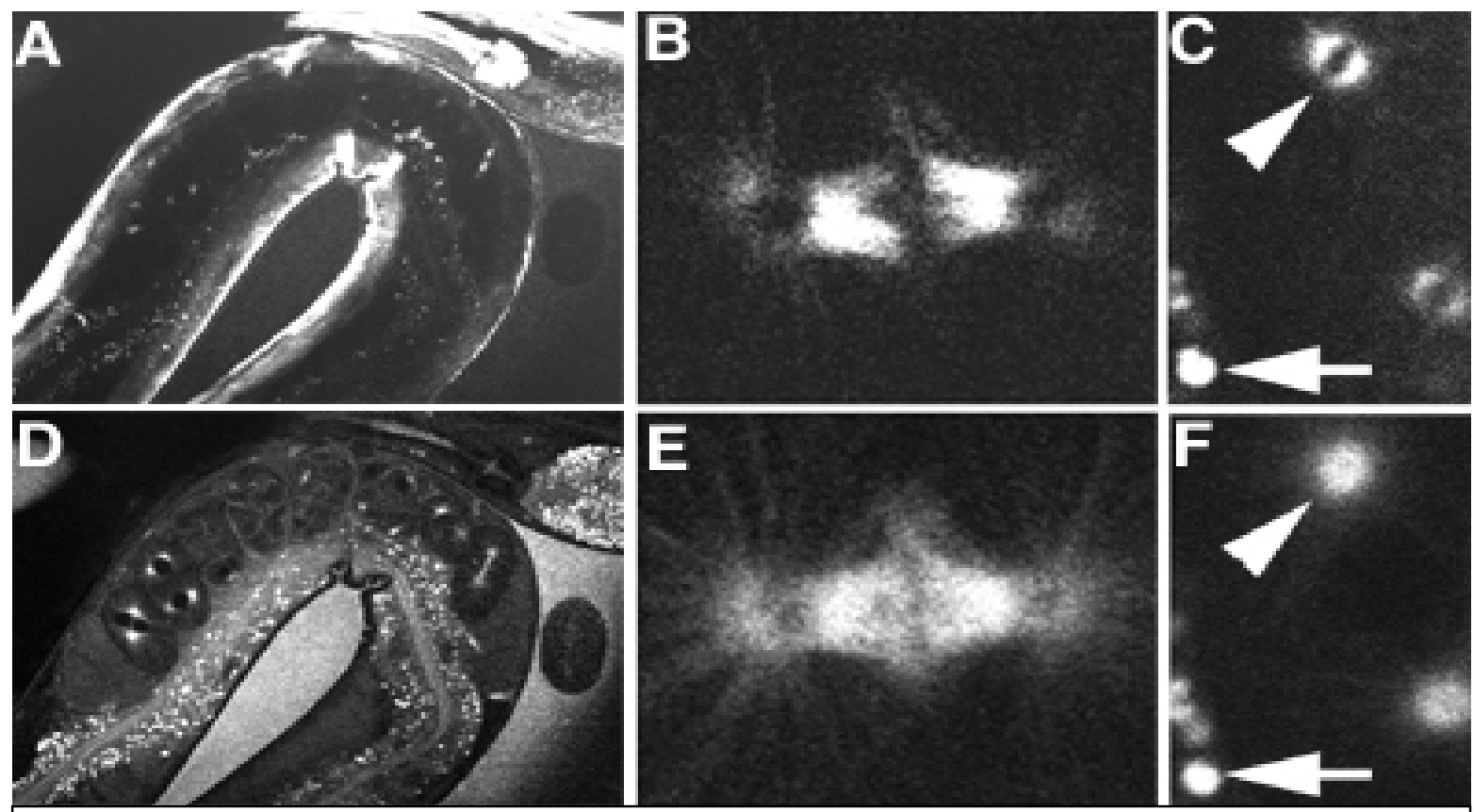

Figure 1. Non-linear optical micrographs of microtubule arrays in nematode embryos. SHG (A, B, C) and TPEF (b-tubulin::GFP) (D, E, F) optical sections through early embryos within the uterus of live $C$. elegans. $(\mathrm{B}, \mathrm{E})$ an embryo shown at higher magnification during the first mitosis. Scale bar $=5 \mu \mathrm{m}$. In $(\mathrm{C}, \mathrm{F})$ two interphase centrosomes in early embryonic cells shown at high magnification (arrowheads in $\mathrm{C}$ and F). SHG-bright granules in the intestine of the mother are indicated (arrow in C).
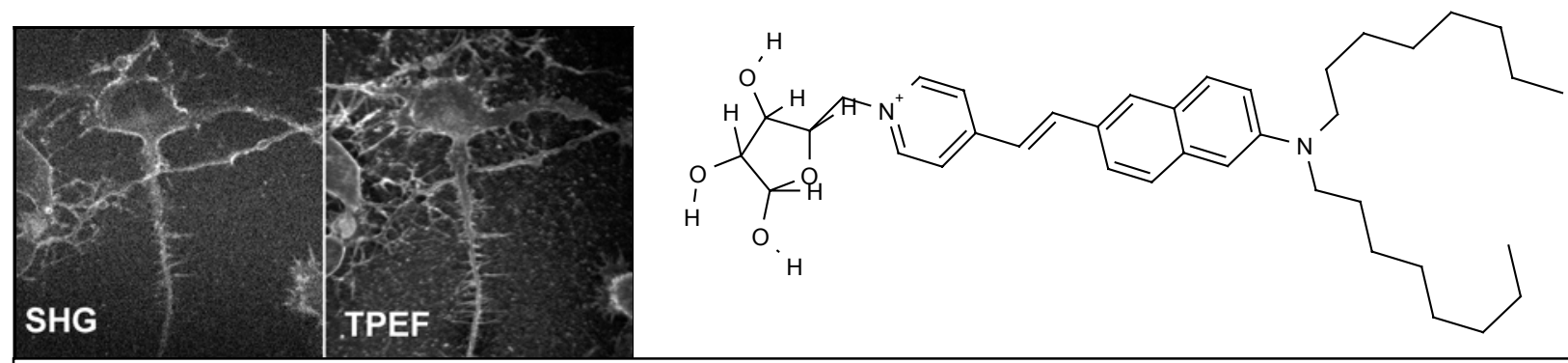

Figure 2. Imaging with chiral styryl dyes. The non-linear optical images of a neuronal cell on the left were obtained after staining the cell membrane with the dye whose structure is shown on the right. The SHG intensity is twice that found for similar achiral styryl dyes. 